\title{
THE USE OF FAST NEUTRON DETECTION FOR MATERIALS ACCOUNTABILITY
}

\author{
L. F. NAKAE, G. F. CHAPLINE, A. M. GLENN, P. L. KERR, K. S. KIM, S. A. OUEDRAOGO, \\ M. K. PRASAD, S. A. SHEETS, N. J. SNYDERMAN, J. M. VERBEKE, R. E. WURTZ
}

Lawrence Livermore National Laboratory, Livermore, California, USA Nakae1@llnl.gov

Published 25 February 2014

\begin{abstract}
For many years at LLNL, we have been developing time-correlated neutron detection techniques and algorithms for applications such as Arms Control, Threat Detection and Nuclear Material Assay. Many of our techniques have been developed specifically for the relatively low efficiency (a few percent) inherent in man-portable systems. Historically, thermal neutron detectors (mainly ${ }^{3} \mathrm{He}$ ) were used, taking advantage of the high thermal neutron interaction cross-sections, but more recently we have been investigating the use of fast neutron detection with liquid scintillators, inorganic crystals, and in the near future, pulse-shape discriminating plastics that respond over 1000 times faster (nanoseconds versus tens of microseconds) than thermal neutron detectors. Fast neutron detection offers considerable advantages, since the inherent nanosecond production timescales of fission and neutron-induced fission are preserved and measured instead of being lost in the thermalization of thermal neutron detectors. We are now applying fast neutron technology to the safeguards regime in the form of high efficiency counters. Faster detector response times and sensitivity to neutron momentum show promise in measuring, differentiating, and assaying samples that have modest to very high count rates, as well as mixed neutron sources (e.g., Pu oxide or Mixed $\mathrm{Cm}$ and $\mathrm{Pu}$ ). Here we report on measured results with our existing liquid scintillator array and promote the design of a nuclear material assay system that incorporates fast neutron detection, including the surprising result that fast liquid scintillator becomes competitive and even surpasses the precision of ${ }^{3} \mathrm{He}$ counters measuring correlated pairs in modest $(\mathrm{kg})$ samples of plutonium.
\end{abstract}

Keywords: Nuclear instrumentation; fission; fission chain; fast neutron detection; time-correlated particle detection; special nuclear material detection; assay.

PACS Numbers: 24.60.Ky, 24.75, 25.85.Ca, 25.85.Ec, 28.20.Pr, 29.40.Mc

\section{Introduction}

The low natural background rates and the penetrating nature of neutron radiation make neutron detection (particularly time-correlated neutrons) a good method for quantifying and accounting for large amounts of special nuclear material (SNM) capable of neutron

This is an Open Access article published by World Scientific Publishing Company. It is distributed under the terms of the Creative Commons Attribution 3.0 (CC-BY) License. Further distribution of this work is permitted, provided the original work is properly cited. 
induced fission and fission chains. Fission is one of the few natural processes that produce time-correlated neutrons - the others are spallation-type processes, like (n,xn) and cosmic induced background - that have low but measurable rates in common terrestrial material. The high rates of most transuranic spontaneous fission sources (like $\mathrm{Pu}$ ) of even a gram or less usually swamp any cosmic induced background rate (in comparison, $\mathrm{kg}$ quantities of natural uranium produce neutrons only on the same order as that of the cosmic background). All other sources of neutrons are generally from alpha-n production (energetic alpha particles fusing with light elements) that generate single neutrons that will be uncorrelated in time. The exploitation of the time-correlated neutron flux from the spontaneous fission of ${ }^{240} \mathrm{Pu}$ is what is used for materials accounting through the world.

\section{Nuclear Material Accounting}

Historically, the standard method employed for the material accounting of plutonium masses is to measure the time-correlated neutron flux from a given sample of material. In principle, one can pick any number of ways to determine the level of time-correlation. In practice, almost all methods employ the measurement of neutron counts over a fixed period of time, that time chosen to be appropriate for the instrument employed. The bin can either be randomly triggered or triggered by a measured neutron. The statistics of these measured bins then can be summed and compared to what would have occurred from a random distribution and from the difference and level of correlation, a quantity determined.

The standard instrument roundly employed around the world is a high efficiency neutron multiplicity counter (NMC). This is essentially a large block of polyethylene moderator with several rows of ${ }^{3} \mathrm{He}$ tubes centered about a chamber where a sample can be inserted; see Fig. 1(a). The detector completely absorbs all the fission spectra (and lower) energy neutrons placed in the sample well, either by thermal capture in the moderating polyethylene or in the ${ }^{3} \mathrm{He}$ where they are counted, and is isolated from external neutrons by a layer of cadmium. In the standard measurement method, the socalled Shift-Register Technique, measurements are made by opening a $32 \mu$ s bin width (which is on the order of the NMC average thermalization/capture time in the polyethylene $/{ }^{3} \mathrm{He}$, which was in fact the NMC $\sim 26 \mu$ s for the one used here) after each measured neutron, and a correction for random (accidental) correlations is made by subtracting a second $32 \mu$ s bin opened $3 \mathrm{~ms}$ after the original bin was triggered. The $3 \mathrm{~ms}$ time was considered long compared to the true neutron correlation detector constant time, and therefore any neutrons measured in that bin would be purely random. This method was easily implemented with a serial gate structure that was readily available 50-60 years ago when plutonium accountability was first needed. The method has proven to be very robust and has been the standard method of plutonium accountability ever since. ${ }^{1}$

Generally, only the pairs correlations (second moment) are used for the actual quantification of a sample, and assumptions are made about the efficiency of the NMC and the multiplication of the sample. The accounting relies on measured net pairs being 
consistent with what it is supposed to be. However, in principle, the higher order correlations (triples, quadruples, etc.) can be used to either show consistency or be solved in order to determine an unknown sample. The method breaks down when the flux becomes high enough that the random correlations measured in the second bin (triggered $3 \mathrm{~ms}$ after the initial detection) becomes significant compared to the original bin. Then the subtraction ends up cancelling out the significance of the measurement. In practice, for a $32 \mu \mathrm{s}$ bin, this begins to occur somewhere with a flux around $10^{5}-10^{6}$ neutrons per second but also is strongly dependent upon the significance of the time correlations being measured (multiplication and nbar of the fissioning material).

Here we propose the use of a fast neutron detection system, e.g., employing liquid scintillator (LS) with pulse shape discrimination (PSD), as a possible alternative to a ${ }^{3} \mathrm{He}$ thermal based system. Even though LS detectors relying on neutron detection through proton recoil have intrinsically low efficiency, the inherent faster timing of the signal allows for much shorter measurement binning and therefore a much lower rate of random correlations. This makes it an attractive method for assaying nuclear material especially material with relatively high neutron fluxes. Figure $1(b)^{2}$ is one arrangement of a current array we have working at LLNL that has approximately a $2 \pi$ solid angle and a roughly 5-7\% true efficiency for fission neutrons produced in the middle of the approximately cylindrical array. ${ }^{3}$

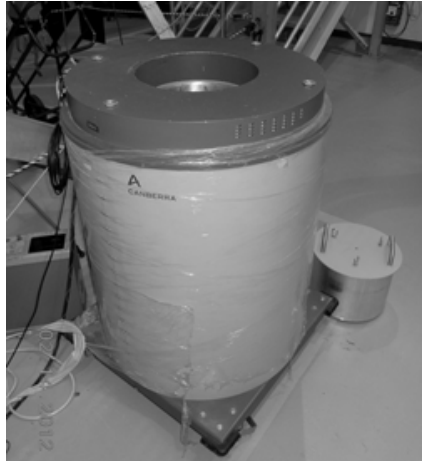

(a)

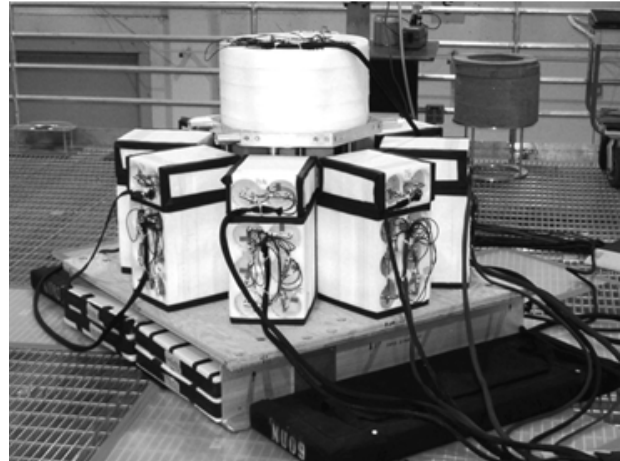

(b)

Fig. 1. (a) High Efficiency Well Counter (3He) and (b) Liquid Scintillator Array (fast neutron array with PSD, approximately $2 \pi$ solid angle).

For the PSD, we calibrate on neutrons and gammas from a ${ }^{252} \mathrm{Cf}$ source and compute a particle ratio of tail-to-total light, with the head-to-tail cutover optimized as described in Ref. 4. We fit Gaussians to the neutron and gamma ensembles. We choose the particletype threshold using these Gaussians, adjusting our rate of gammas misidentified as neutrons per gamma (sometimes called "gamma rejection rate or GRR") at 5-sigma, i.e., no more than 1 out of $3.5 \times 10^{6}$. Under high background gamma rates, the neutron ensemble is contaminated by piled-up gamma events. Many of these pulses are flagged by the presence of two peaks and rejected from our analysis. Regarding the un-flagged 
pileups, we estimate that at particle rates causing $1 \%$ pileup, the Gaussian with a 5-sigma cut is still a reasonable GRR estimator. For our cells and electronics, $1 \%$ is about $10^{4}$ counts per channel. For reference, $\mathrm{kg}$ quantities of plutonium shielded with $3 / 8$ inch of $\mathrm{Pb}$ are well below this count rate.

\section{Comparisons of Data from a NMC and the LLNL Liquid Scintillation Array}

Modern electronics allows for list mode data collection, which is ideal for laboratory development and algorithm testing. We were fortunate to have been able to borrow an NMC from our collaborators at the Atomic Weapons Establishment (AWE, Aldermaston, UK) that was fitted in such a way that we could collect list mode data from an NMC. With the list mode data stream we could mimic the structure traditionally employed in the NMC, but because we have developed here at LLNL a different method of analysis, we show here data displayed in our fashion where we use random gates and account for the random correlations by using a theoretical prediction of random correlations calculated from the bin width and the measured count rate and plot them against each other to show the significance of correlation.

In displaying our data, we prefer to plot the data for measurement with the $\mathrm{X}$ axis being the multiplicity (number of neutrons measured for a particular binning) vs. the number of times we measured that multiplicity for as many measurements as were taken. We can either have the $\mathrm{Y}$ axis simply be that number of times we measured a particular multiplicity, or for the case of the random bin triggering, turn it into a probability distribution by dividing the $\mathrm{Y}$ axis by the number of times we did the measurement. For triggered binning, like the shift register method, a similar displaying method can be performed, but it does not have as straight forward an interpretation as a randomly triggered probability distribution. We plot the data (circles in this paper) displayed in this fashion vs. an easily calculated prediction of what the distribution would be if the source were random (Poisson) distribution (triangles in this paper), and any significant deviation of a wider distribution (larger variance) is an indication of a correlated neutron source. We also like to compare the measured distributions to a second theoretically generated distribution calculated from a simple abstracted model where there is only a source term, multiplying isotope, multiplication ( $\mathrm{M}=1 / 1-\mathrm{Keff}$ ), an alpha ratio ( alpha-n neutron fraction compared to the source), a detector efficiency and a time constant for the correlation.

In Fig. 2 we can compare data taken with a modest strength ${ }^{252} \mathrm{Cf}$ source (source strength $\sim 7.8 \times 10^{4} \mathrm{n} / \mathrm{s}$; random triggering) with the NMC to data taken with our fast Liquid Scintillator Array (LSA). Figure 2(a) is $32 \mu \mathrm{s}$ bin width data taken with the thermal detecting NMC, and Fig. 2(b) is $50 \mathrm{~ns}$ bin width data taken with the LSA. The data in Fig. 2(a) are circles and the triangles are the prediction of what the data would look like if the source was random (given the same count rate and bin width). The level of deviation (wider variance) of the data from the random (Poisson) distribution (shaded to aid the eye) is indicative of the level of correlation in the neutron data. A very large deviation is indicative of a highly multiplying source. In both data sets the correlation 
level is strong and there is no difficulty in determining the strength of the Cf source. This is due to the relatively large average number of neutrons generated per spontaneous fission of ${ }^{252} \mathrm{Cf}$ (nbar $=3.787$ ). It is important to note that the LSA data set shows a comparable level of correlation even though it is a factor of 9 or 10 less efficient than the NMC. This is because the LSA can use a much shorter bin 1000 times smaller and so there are many fewer random correlations. While the data sets are comparable, it is true that here the significance is still larger (measured error is smaller) for the $50 \%$ efficient LMC than the $6 \%$ efficient LSA. Again this is due to the relatively modest source strength $\left(\sim 7.8 \times 10^{4} \mathrm{n} / \mathrm{s}\right)$ and the high level of neutron multiplicity in the spontaneous fission of ${ }^{252} \mathrm{Cf}$.

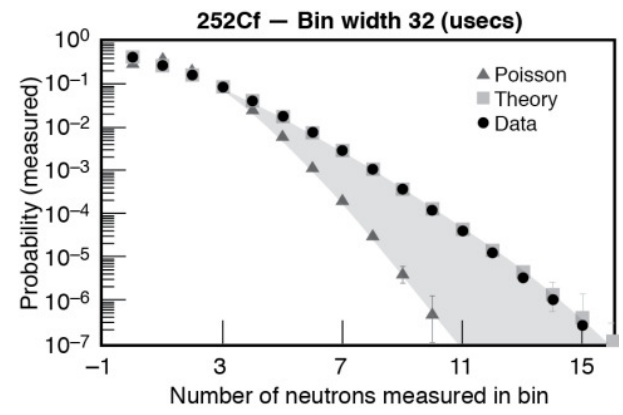

(a)

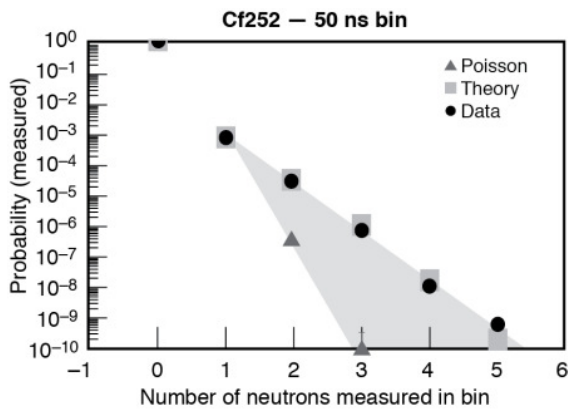

(b)

Fig. 2. Data (random triggering) from a ${ }^{252} \mathrm{Cf}$ source with (a) a $50 \%$ efficient ${ }^{3} \mathrm{He} \mathrm{NMC}, 32 \mu \mathrm{s}$ bin and (b) a $6 \%$ efficient Liquid Scintillator Array, $50 \mathrm{~ns}$ bin. The shaded area illustrates the significance of correlation of data (circles) from a prediction of a random (Poisson) distribution (triangles) with the same count rate and bin width.

A more difficult problem is to measure the correlations in a stronger source with a lower level of multiplicity and a higher level of random correlations. An example of such a source of very high interest is a kilogram quantity of plutonium oxide. Spontaneous fission in plutonium is mostly from ${ }^{240} \mathrm{Pu}$, which is a relatively strong neutron source $(\sim 1000 \mathrm{n} / \mathrm{s}$-gm) with a very modest multiplicity (nbar $=2.16)$, and Pu oxide is very low density and does not multiply very much. Pu oxide also comes with alpha-n neutrons that add random neutrons to the measurement in significant quantities. Pure weapon's grade $\mathrm{Pu}$ oxide has nearly as many alpha-n neutrons (from the $\mathrm{Pu}$ and Am alpha particles on ${ }^{18} \mathrm{O}$ ) as from spontaneous fission. Oxides can also have other contaminants like carbon, fluorine, beryllium or aluminum from processing that can also make alpha-n neutrons.

In Fig. 3 we show data taken on a $\sim 1.7 \mathrm{~kg}$ of Pu oxide with an alpha-n ratio of $\sim 2.5$ ). The neutron source strength was $\sim 3.5 \times 10^{5} \mathrm{n} / \mathrm{s}$, and the multiplication was about 1.2. One can see that for the NMC (Fig. 3(a)), the significance of the variance difference between the data and a Poisson distribution is much reduced. In fact one must go out to the nineneutron multiplet to see the difference by eye. Since the pairs (and even triples) are dominated by the first few multiplets (note the $\mathrm{Y}$ axis is a log scale), there is not much signification to the first few moments compared to a random source. Now looking at the 
same sample measured with the LSA, shown in Fig. 3(b), we can see the effect of using a much shorter bin of $50 \mathrm{~ns}$, which is possible because we are detecting fast neutrons and do not have to wait for the thermalization time required to detect neutrons with a ${ }^{3} \mathrm{He}$ NMC. Since the bin width is almost 1000 times shorter, there is a huge suppression of random neutrons, and there are almost no expected random correlations even in the $2^{\text {nd }}$ moment. It is evident that the fast detector system, even being $1 / 10$ as efficient, has made a more precise measurement. It is also worth pointing out that if one were to attempt to find a solution to the unknown system using the higher order correlations in the data set, the solutions for the 50\% NMC are highly degenerate because of the high level of random (accidental) correlations, which destroy the significance of the measured multiplets. This means that the highest possible efficiency thermal detector never could resolve the masses of an unknown object with much certainty. The fast neutron LSA has no trouble resolving these details because there are little or no "accidentals" within a short $50 \mathrm{~ns}$ bin.

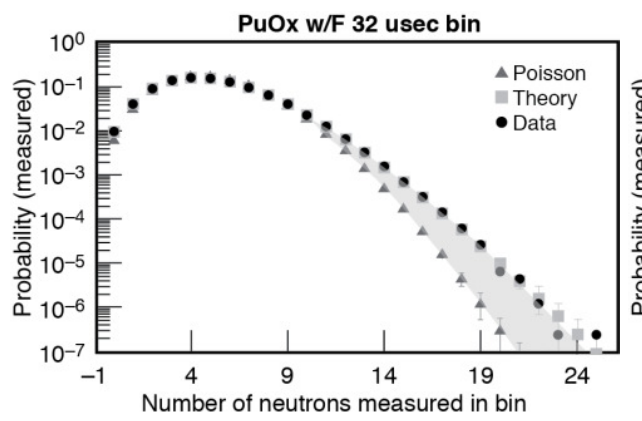

(a)

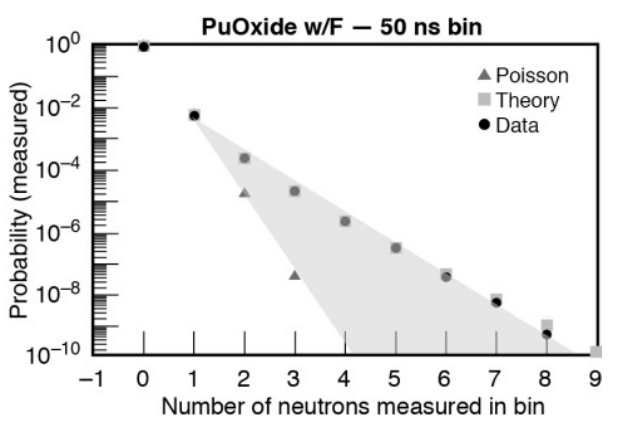

(b)

Fig. 3. Data from a Pu Oxide sample: (a) a 50\% efficient NMC and (b) the LLNL Liquid Scintillator Array.

\section{Summary: The Use of Fast Neutron Detectors in Safeguards}

The much lower random correlation rates in the faster liquid scintillator versus thermal ${ }^{3} \mathrm{He}$ and the better precision for higher fluxes has profound implications for nuclear safeguards accountability. The crossover point is of course source and detector dependent, depending on how significant the source correlations actually are. What has been implied by the measurements shown in Figs. 2 and 3 is illustrated a slightly different way in Fig. 4. For our current $\sim 5 \%$ efficient liquid scintillator array versus a $50 \%$ efficient ${ }^{3} \mathrm{He}$ well counter, this occurs at about $10^{6} \mathrm{n} / \mathrm{s}$ for ${ }^{252} \mathrm{Cf}$ (Fig. 4(a)) and $7 \times 10^{4} \mathrm{n} / \mathrm{s}$ for pure ${ }^{240} \mathrm{Pu}$ samples (Fig. 4(b)).

Crossovers are lower for sources in oxide form or when including the amount of alpha-n random neutrons, which degrades the significance of the correlated spontaneous fission sources. This means that an efficient fast neutron detector like our LSA is a better choice for the accountability of $\mathrm{kg}$ quantities of plutonium, particularly in oxide form, than a ${ }^{3} \mathrm{He}$ system, even if the ${ }^{3} \mathrm{He}$ were available as is the case for accounting for MOX 


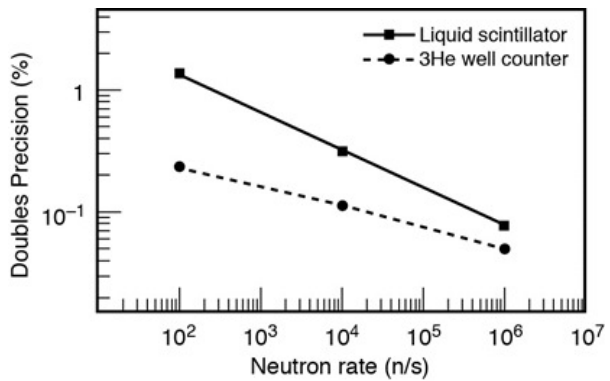

(a)

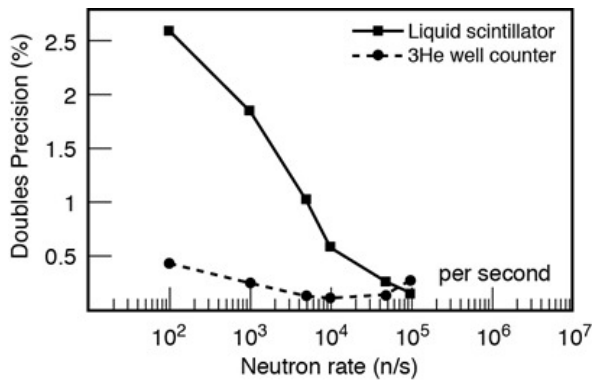

(b)

Fig. 4. Pairs coincidence precision as a function of neutron rate for (a) measured ${ }^{252} \mathrm{Cf}$ sources and (b) simulated ${ }^{240} \mathrm{Pu}$.

fuel. This is with $\sim 5 \%$ detector system that we are currently employing. One can see that our system is a $\sim 2 \pi$-solid-angle detector, and simply by better filling in the available solid angle, we could gain 50-100\% more solid angle - meaning even better precision than demonstrated here.

It may be also possible, with further development, to help with a currently unsolved problem of differentiating between complicated samples containing different fractions of spontaneous fission sources, for example $\mathrm{Cm}$ versus $\mathrm{Pu}$ or $\mathrm{Cf}$, as they all of produce a different average number of neutrons per fission. However, more importantly, they will come with differing amounts of other fissionable material, and therefore by subjecting the samples to varying amounts of moderation and reflection, one may be able to discern relative amounts of fissionable material within a sample.

In the longer term, plastic scintillators doped for pulse-shape discrimination (PSD) (currently under development at LLNL) can be expected to have even better stability and field performance. The current fast neutron detectors all employ scintillation with PSD. The Achilles heel of such detectors is that they are all sensitive to gamma rays as well as neutrons. Often the gamma ray flux for objects of interest can be 1000 to 10,000 times the neutron flux, and so the true limiting factor for the efficiency of these fast systems is the gamma-ray flux. While it is true that high $\mathrm{Z}$ shielding can certainly help in some cases, MeV gamma rays are difficult to block completely, and when they are present in high flux as in the problem of quantifying large amounts of spent fuel, where one is dominated by the beta delay gamma rays in the fission fragments, there is no way the detector can be effectively shielded from the gamma ray flux and therefore fast neutron/gamma ray sensitive scintillators will not be the solution. However, if a sufficiently efficient fast neutron detector that is insensitive to gamma rays can be constructed, this would be the holy grail, and then even the spent fuel problem can be addressed. Some current developments show some promise, ${ }^{5}$ but the efficiency is the question.

In any event, it is evident that the advent of modern fast digital electronics has made it possible to take advantage of the inherent advantages of nanosecond timing of neutron 
correlations, which is the proper time-scale to measure these correlations. It is time to revisit the standard methods for performing safeguards accountability employing only the highest possible efficiency with thermal ${ }^{3} \mathrm{He}$ detectors and, especially in the limiting regions of weak signals and high fluxes, consider using fast neutron technology to improve precision and expand the areas that can be investigated.

\section{Acknowledgments}

This work has been performed under the auspices of the U.S Department of Energy by Lawrence Livermore National Laboratory under Contract DE-AC52-07NA27344.

\section{References}

1. A. D. Dougan, N. J. Snyderman, L. F. Nakae, D. D. Dietrich, P. L. Kerr, T. F. Wang, W. Stoeffl, S. Friedrich and L. Mihailescu, New and Novel Nondestructive Neutron and GammaRay Technologies Applied to Safeguards, in 2007 JAEA-IAEA Workshop on Advanced Safeguards Technology for the Future Nuclear Fuel Cycle, \#4A.2 (Tokai-mura, Japan, 2007).

2. P. Kerr, M. Rowland, D. Dietrich, W. Stoeffl, B. Wheeler, L. Nakae, D. Howard, C. Hagmann, J. Newby and R. Porter, Nucl. Instr. Meth. B 261, 347 (2007).

3. L. F. Nakae, P. L. Kerr, R. J. Newby, M. K. Prasad, M. S. Rowland, N. J. Snyderman, J. M. Verbeke and R. E. Wurtz, Recent Developments in Neutron Detection and Multiplicity Counting with Liquid Scintillator, in $2^{\text {nd }}$ Japan IAEA Workshop on Advanced Safeguards Technology for the Future Nuclear Fuel Cycle (Tokai, Japan, 2010), https://e-reportsext.llnl.gov/pdf/384910.pdf.

4. I. A. Pawelczak, S. A. Ouerdraogo, A. M. Glenn, R. E. Wurtz and L. F. Nakae, Nucl. Instr. Meth. Phys. Res. A 711, 21 (2013).

5. R. Chandra, G. Davatz, H. Friederich, U. Gendotti and D. Murer, Fast neutron detection with pressurized ${ }^{4} \mathrm{He}$ scintillation detectors, in $2^{\text {nd }}$ International Workshop on Fast Neutron Detectors and Applications (Ein Gedi, Israel, 2011). 